\title{
CLINICAL-RADIOLOGICAL CORRELATION OF ORBITAL CYSTICERCOSIS
}

\section{Ophthalmology}

Palak Gupta

Vikrant Mahajan*

\section{Anchal Gupta}

Resident, Department of Ophthalmology, Government Medical College, Jammu, Jammu and Kashmir, India.

Senior Resident, Department of Radiodiagnosis and Imaging, Government Medical College, Jammu, Jammu and Kashmir, India. *Corresponding Author

Senior Resident, Department of ENT, SMGS Hospital, Jammu, Jammu and Kashmir, India.

\section{ABSTRACT}

Background: Orbital cysticercosis is an infectious cause of preventable blindness not uncommon in India. It is a parasitic infestation caused by Cysticercus cellulosae, which is the larval form of Taenia solium. The aim of our study was to evaluate the role of the ultrasonography and CT scan in detecting ocular and adnexal cysticercosis.

Materials and methods: This hospital based retrospective study was conducted in the department of Ophthalmology and Radio-diagnosis of GMC Hospital Jammu from July 2017 to January 2020. A total of 12 patients with suspected diagnosis of ocular and adnexal cysticercosis were studied with the help of ophthalmic examination, serology profile, ultrasonography and CT imaging.

Results: Our study included 12 patients with male to female ratio 2:1. Majority of the patients had unilateral presentation with only two having bilateral eye involvement. The most common site of lodgement of orbital cysts was seen in the subconjunctival space in 4 patients ( $33 \%)$, followed by $2(16 \%)$ in eyelid, $2(16 \%)$ in extraocular muscles, $2(16 \%)$ in posterior segment, $1(8 \%)$ in optic nerve and $1(8 \%)$ retro-orbital space. Indirect ophthalmoscopy in one case showed a free floating cyst in the vitreous cavity while in two other patients a translucent cyst with an intracystic whitish spot suggestive of scolex was demonstrated.

Conclusion: On the basis of present study, it can be concluded that the diagnosis of orbital cysticercosis is usually accomplished by high degree of clinical suspicion, ophthalamoscopic demonstration of the larval worm and the characteristic features on imaging studies. CT scan can be helpful in assessing extraocular cysticercosis, neurocysticercosis and ancillary findings, if any.

\section{KEYWORDS}

Cysticercosis, scolex, neurocysticercosis, ultrasound, CT scan

\section{INTRODUCTION}

Orbital cysticercosis is an infectious cause of preventable blindness not uncommon in India ${ }^{[1]}$. It is a parasitic infestation caused by Cysticercus cellulosae, which is the larval form of Taenia solium. The first case of a live ocular cysticercosis was reported by Soemmering ${ }^{[2]}$ in 1829. Orbital cysticercosis shows predilection for children and young adults with no sex predilection ${ }^{[3,4]}$. Human cysticercosis predominantly affects the central nervous system causing neurocysticercosis and also the eye causing ocular cysticercosis ${ }^{[5]}$. The manifestations of cysticercosis reflect two distinct processes: (i) the local inflammatory response induced by the parasite; and (ii) the local effect of the space-occupying lesions. The onset of symptoms also depends on the site of cyst lodgement in the orbit, so irrespective of the stage of cyst evolution, the tighter and limited space of the orbit may lead to early onset of symptomatology merely due to a mass effect. B-scan ocular ultrasonography (US) is used as a technique to detect intraocular cysticercosis in patients. It has several advantages like the accessibility, opportunity, cheapness, monitoring and not being invasive in critical patient. However any orbital adnexal involvement is better seen on cross sectional imaging techniques like CT scan and MRI. Therefore, the aim of our study was to evaluate the role of the ultrasonography and CT scan in detecting ocular and adnexal cysticercosis.

\section{MATERIALS AND METHODS}

This hospital based retrospective study was conducted in the department of Ophthalmology and Radio-diagnosis of GMC Hospital Jammu from July 2017 to January 2020. A total of 12 patients with suspected diagnosis of ocular and adnexal cysticercosis were studied. The age, gender and mode of presentation were recorded. Detailed ophthalmologic examinations including visual acuity, slit lamp biomicroscopy, diplopia charting, direct and indirect ophthalmoscopy were performed for each eye. A general physical examination was performed in all the cases to detect cysticercus if present in other parts of the body. The serum from each patient was tested for cysticercus antibodies by enzyme linked immunosorbent assay (ELISA). The ELISA kit used was IgM ELISA. Other routine laboratory investigations included routine stool microscopy for intestinal parasites, ESR, total and differential leucocyte count, and absolute eosinophil count. Ultrasonography (B-scan) was performed in all cases. High frequency linear USG probe was used for orbital examination. The subjects were examined in the supine position.
Conductive US gel was placed over a closed eyelid. The scan was performed with the patient fixing in primary gaze. A computerized tomography (CT) scan of the brain and orbit was carried out to rule out the possibility of neurocysticercosis and to supplement the diagnosis of ocular cysticercosis respectively. A diagnosis of ocular cysticercosis was made based on ultrasonographic or CT evidence of cyst with or without scolex and serum positivity for antibodies by ELISA.

\section{RESULTS}

Our study included 12 patients, out of whom there were 8 male (67\%) and 4 female $(33 \%)$ patients. The male to female ratio was $2: 1$. Majority of the patients had unilateral presentation with only two having bilateral eye involvement. Majority of the patients had age of the patients from 12 years old to 40 years old. Only two patients had age more than 40 years. The intraocular pressures of all subjects were normal. Table 1 shows demographic details of patients. Nine patients $(75 \%)$ presented with periorbital swelling, the most common sign in our study; with loss of vision (67\%), irritation of eyes (67\%), pain $(58 \%)$, proptosis as other common signs. In addition, diplopia and restriction of ocular motility were also relatively frequent. Figure 1 shows clinical presentation of patients.

Table 1: Demographics of patients

\begin{tabular}{|c|l|}
\hline Gender & 8 \\
\hline Male & 4 \\
\hline Female & \\
\hline Laterality & 10 \\
\hline Unilateral & 2 \\
\hline Bilateral &
\end{tabular}

Clinical presentation of patients

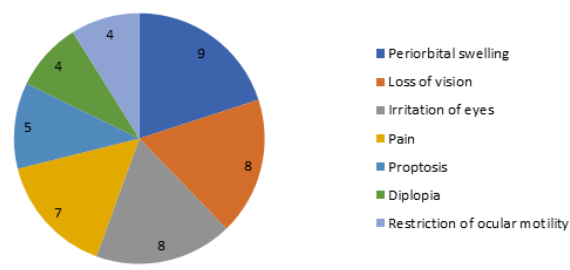

Figure 1: Signs and Symptoms of patients 
In our study, ultrasonography displayed the characteristic picture of a sonolucent area with well-defined anterior and posterior margins. The presence of a central echodense scolex was seen in $10(83 \%)$ majority of cases. CT scan of brain and orbit was performed in 6 cases. It was seen as normal in the three cases studied. One of the patient showed cyst with no definite scolex within superior rectus extra-ocular muscle. Two patients revealed multiple ring enhancing lesion scattered in supra as well as infatentorial brain parenchyma with multiple orbital cysts. Indirect ophthalmoscopy in one case showed a free floating cyst in the vitreous cavity (5 disc diameters in size) while in another patient a translucent cyst with a intracystic whitish spot suggestive of scolex was demonstrated in optic nerve head region. Lodgement of cysts was seen in the subconjunctival space in 4 patients $(33 \%)$, followed by $2(16 \%)$ in eyelid, $2(16 \%)$ in extraocular muscles, $2(16 \%)$ in posterior segment, $1(8 \%)$ in optic nerve and $1(8 \%)$ retro-orbital space.

\section{Table 2: Location of cysticercosis}

\begin{tabular}{|l|l|}
\hline Location & No. of patients \\
\hline Subconjunctival & 4 \\
\hline Eyelid & 2 \\
\hline Extraocular muscles & 2 \\
\hline Posterior segment & 2 \\
\hline Optic nerve & 1 \\
\hline Retro-orbital space. & 1 \\
\hline
\end{tabular}

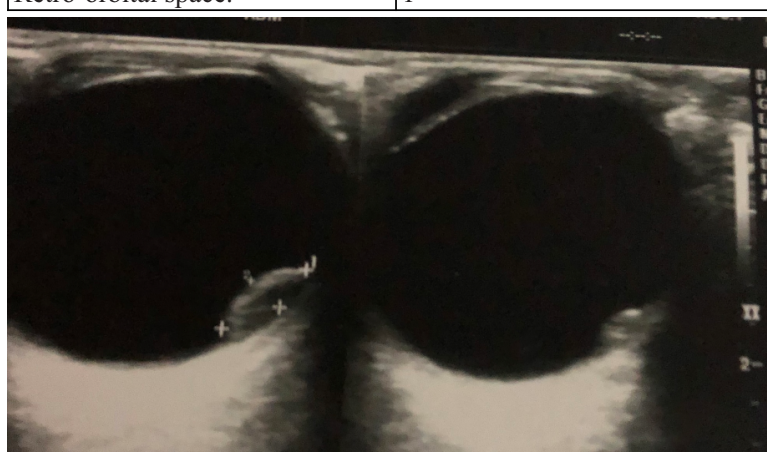

Figure 2: B-scan ultrasound picture showing a reveals a well-defined cystic lesion with partially clear contents along the nasal side of ocularglobe.

\section{DISCUSSION}

Soemmering reported the first case of ocular cysticercosis in 1830 . The larva was demonstrated and extracted by Schott in $1836^{[5]}$. The cysticercus reaches the orbit through the choroid vessels, having primitive subretinal location. Inside the orbit it can induce inflammatory reaction and blindness in $8 \%$ of patients ${ }^{[6]}$. While the most common site of localisation reported in Western studies is the posterior, in the Indian literature the ocular adnexa is the most common site ${ }^{[7-9]}$. The common clinical complains are periocular swelling, proptosis, ptosis, pain, diplopia, restriction of ocular motility, strabismus, decreased vision, lid edema and orbital cellulitis like clinical picture. In cases of extraocular muscle involvement, superior rectus muscle is the most common site ${ }^{[10]}$. Subconjunctival presentation could be a secondary stage in those cases in which the cyst may have extruded from the primary extra ocular muscle site ${ }^{[11]}$. It has to be differentiated from other benign and malignant conditions presenting as ocular mass. The differential diagnosis of orbital cysticercosis includes idiopathic myositis, tumours or metastasis, muscle abscess or haematoma, and other parasitic infections like hydatid cyst.

On indirect ophthalmoscopic examination, a live cyst can be seen as a translucent white cyst with dense white spot formed by the invaginated scolex with typical undulating movements. Serodiagnostic test is helpful but not specific. Laboratory studies are of limited value in intraocular cysticercosis. Eosinophilia is usually absent unless there is widespread dissemination of the parasite. Serological tests lack sensitivity ${ }^{[12]}$. The indirect haemagglutination test shows cross reactivity between cysticercosis and echino coccosis. The diagnosis of orbital cysticercosis can be made clinically, however radiological imaging like ultrasonography of the posterior segment and orbit, CT scan or MRI of the brain helps to rule out other sites of involvement by the cyst- both ocular as well as systemic, especially neurocysticercosis. In our study, 12 patients with clinically suspected orbital cysticercosis were evaluated with B-mode USG and CT scan.
Majority of the patients in our study had unilateral involvement. We found no predilection for either sex as in most other studies ${ }^{[8,13]}$. The majority of our patients were less than 40 years of age. Reddy et al reported that $90 \%$ of their patients were less than 15 years of age ${ }^{[9]}$ Malik et al reported $68 \%$ in the age group of $10-30$ years ${ }^{[8]}$. In our study, on ultrasonography lodgement of cysts was seen in the subconjunctival space in 4 patients $(33 \%)$, followed by $2(16 \%)$ in eyelid, $2(16 \%)$ in extraocular muscles, $2(16 \%)$ in posterior segment, $1(8 \%)$ in optic nerve and $1(8 \%)$ retro-orbital space. The posterior segment is more affected in western countries, whereas in India the cysts are more often subconjunctival ${ }^{[13]}$. In a series on ocular cysticercosis from India, Reddy PS et al reported $60 \%$ of the patients had subconjunctival Cysticerci ${ }^{[9]}$. Rath S concluded the most common locations of cyst was in the anterior orbit in $69 \%$ of cases, subconjunctival space in $24.6 \%$, posterior orbit in $5.8 \%$, and the eyelid in $0.6 \%$ of cases ${ }^{[10]}$. Multiple cysts may develop in the same eye ${ }^{[14]}$

Orbital imaging studies, such as CT or MRI, though are better means of orbital cysticercosis, USG can be used as a screening method in settings where these expensive imaging machines are not readily available. USG can be helpful to the practicing clinician in diagnosing orbital cysticercosis using a non-invasive tool at bedside. Our study concluded that the diagnosis of cysticercosis is made by the clinical findings and supported by other tests like serological tests (ELISA), USG and CT scan. Serial B-scan ocular ultrasonography or CT scanning of the orbit helps to follow the resolution of the cyst, which is recognized by the disappearance of the scolex.

\section{CONCLUSION}

On the basis of present study, it can be concluded that orbital and adnexal cysticercosis has a wide spectrum of clinical manifestations. It can masquerade as any of the subacute, acute or chronic inflammatory or cystic lesions of the orbit and adnexa, particularly in young patients from endemic areas of cysticercosis. CT scan is helpful in assessing extraocular cysticercosis, neurocysticercosis and ancillary findings, if any. Diagnosis of ocular cysticercosis is usually accomplished by high degree of clinical suspicion, ophthalamological examination and the characteristic features on imaging studies.

\section{REFERENCES}

. David S, Mathai E. Ocular cysticercosis--a review of 25 cases. J Assoc Physicians India 2000;48(7):704-707

2. Sommering ST. Uber einen Cysticercus cellulosae im Men- schlichen Auge. Oken' Iseis $1830 ; 8: 717-718$.

Lombardo J. Subretinal cysticercosis. Optom Vis Sci 2001;78(4):188-194.

4. Pushker N, Bajaj MS, Betharia SM. Orbital and adnexal cysticercosis. Clin Exp Ophthalmol2002;30(5):322 333 .

Duke-Elder S, editors. Cysticercosis, System of Ophthalmology. CV Mosby: St. Louis; 1978.p. 40

6. Silberrt PL, Gubbay SS, Khangure M. MRI findings in a case of neurocysticercosis. Med Silberrt PL, Gubbay SS, K

Sekhar GC,Lemke BN. Orbital cysticercosis. Ophthalmology 1997;104:1599-602.

8. Malik SRK, Gupta AK, Choudhry S. Ocular cysticercosis. Am J Ophthalmol 1968;66:1168

9. Reddy PS, Satyendran OM. Ocular cysticercosis. Am J Ophthalmol 1964;57:664

10. Rath S, Honavar SG, Naik M, Anand R, Agarwal B, Krishnaiah S, Sekhar GC. Orbital cysticercosis: clinical manifestations, diagnosis, management, and outcome. Ophthalmology 2010;117(3):600-605.

11. Sundaram PM, Jayakumar N, Noronha V. Extraocular muscle cysticercosis-a clinical challenge to the ophthalmologists. Orbit 2004; 23(4):255-262.

12. Wadhwa V, Kharbanda P, Rai S. How reliable are serological tests in diagnosis of cysticercosis? Indian J Med Microbiol 2006;24(1):78-79

13. Cano MR. Ocular cysticercosis. In: Ryan SJ, editors. Retina. CV Mosby: St. Louis; 1989. p. 583-7.

14. Kapoor S, Kapoor MS. Ocular cysticercosis. J Pediatr Ophthalmol Strabismus.1978; $15(3): 170-3$ 